\title{
Statistical Term Profiling for Query Pattern Mining
}

\author{
Paul Buitelaar \\ DFKI GmbH \\ Language Technology Lab \\ Saarbrücken, Germany \\ paulb@dfki.de
}

\author{
Pinar Oezden Wennerberg, Sonja Zillner \\ Siemens AG \\ Knowledge Management CT IC 1 \\ Munich, Germany \\ pinar.wennerberg.ext@siemens.com, sonja.zillner@siemens.com
}

\section{Introduction}

Through advanced technologies in clinical care and research, especially the rapid progress in imaging technologies, more and more medical imaging data and patient text data is generated by hospitals, pharmaceutical companies, and medical research. For enabling advanced access to clinical imaging and text data, it is relevant to know what kind of knowledge the clinician wants to know or the queries that clinicians are interested in. Through intensive interviews and discussions with radiologists and clinicians, we have learned that medical imaging data is analyzed - and hence queried - from three different perspectives, i.e. the anatomic perspective addressing the involved body parts, the radiology-specific spatial perspective describing the relationships of located anatomical regions to other anatomical parts, and the disease perspective distinguishing between normal and abnormal imaging features. Our aim is to establish query patterns reflecting those three perspectives that would typically be used by clinicians and radiologists to find patient-specific sets of relevant images.

The context of our work is in the TheseusMEDICO $^{1}$ project on cross-modal image and information retrieval in the medical domain. The focus of the work reported here is on setting up Wikipedia-based corpora of human anatomy and radiology and on obtaining a statistical profile of concepts from three semantic knowledge resources with these corpora: the Foundational Model of Anatomy (FMA), the radiology lexicon RadLex, and a subset of the international classification of disease codes ICD-9 CM. Using this information, we intend to extract relations that are likely to occur between statistically relevant terms and the concepts they express.

The final goal of our work is to derive potential query patterns from the extracted set of relations that can be used in the MEDICO semantic-based

\footnotetext{
${ }^{1} \mathrm{http}: / /$ theseus-programm.de/scenarios/en/medico
}

image retrieval application. For example when restaging head and neck lymphoma, clinicians and radiologists look for information and images that report on essential radiological patterns as "an enlargement in the dimension of the lymph node in the neck". Therefore, within our approach, we aim at establishing hypotheses about possible user queries, i.e. the query patterns that reflect the three perspectives discussed above. Accordingly, an example query pattern might look like this:

\begin{tabular}{|c|c|c|}
\hline $\begin{array}{l}\text { [ANATOMICAL } \\
\text { STRUCTURE] }\end{array}$ & located_in & $\begin{array}{l}\text { [ANATOMICAL } \\
\text { STRUCTURE] }\end{array}$ \\
\hline $\begin{array}{l}\text { [[RADIOLOC } \\
\text { IMAGE]Mod }\end{array}$ & $\begin{array}{l}\text { AND } \\
i s \_a b o u t\end{array}$ & [ANATOMICAL \\
\hline IM & & \\
\hline $\begin{array}{l}\text { [[RADIOLOGY } \\
\text { IMAGE]Modality] }\end{array}$ & $\begin{array}{l}\text { shows_ } \\
\text { symptom }\end{array}$ & $\begin{array}{l}\text { [DISEASE } \\
\text { SYMPTOM] }\end{array}$ \\
\hline
\end{tabular}

Once an initial set of similar patterns has been established in this way, they will be evaluated by clinicians for their validity and relevance.

\section{Corpora}

A central aspect of the query pattern mining task is the statistical analysis of the FMA and RadLex terms in relevant text collections. In this way relevance scores can be assigned to terms that allow to investigate the most likely expressed (and hence queried) relations between them. For this purpose we need access to a representative corpus of texts that at the same time reflects the joint view of anatomy, spatial aspects of radiology and disease that we are targeting. Patient records would be our first choice, but due to strict anonymization requirements these are difficult to obtain. We therefore constructed a corpus based on the Wikipedia Categories Anatomy and Radiology. We then ran all text sections of each corpus through a part-ofspeech tagger (Brants, 2000) to extract all nouns in the corpus and to compute a relevance score (chisquare) for each by comparing anatomy and radiology frequencies with those in the British Na- 
tional Corpus. A next step will be to parse and annotate sentences with predicate-structure information, which may then be used for relation extraction along the lines of (Schutz and Buitelaar, 2005).

\section{FMA Terms}

The statistically most relevant FMA terms were identified on the basis of chi-square scores computed for nouns in each corpus. Single word terms in the FMA and occurring in the corpus correspond directly to the noun that the term is build up of (e.g. the noun 'ear' corresponds to the FMA term ear). In this case, the statistical relevance of the term is the chi-square score of the corresponding noun. In the case of multi-word terms occurring in the corpus, the statistical relevance is computed on the basis of the chi-square score for each constituting noun and/or adjective in the term, summed and normalized over the length of the term. Thus, the relevance value for lymph node is the summation of chi-square scores for 'lymph' and 'node' divided by 2 . In order to take frequency in account, we further multiplied the summed relevance value by the frequency of the term. This assures that only frequently occurring terms are judged as relevant.

\begin{tabular}{|l|r|r|r|}
\hline FMA Term & Freq. & Score & POS \\
\hline lateral & 464 & 338724,00 & JJ \\
\hline anterior & 452 & 314721,00 & JJ \\
\hline artery & 237 & 281961,00 & NN \\
\hline anterior spinal artery & 2 & 219894,33 & JJ JJ NN \\
\hline lateral thoracic artery & 2 & 217815,33 & JJ JJ NN \\
\hline
\end{tabular}

Table 1: top FMA terms in anatomy corpus

\begin{tabular}{|l|r|l|r|}
\hline \multicolumn{1}{|c|}{ FMA Term } & Freq. & Score & \multicolumn{1}{l|}{ POS } \\
\hline artery & 65 & 6724,00 & NN \\
\hline coronary artery & 17 & 5284,00 & JJ NN \\
\hline small bowel & 11 & 4651,79 & JJ NN \\
\hline renal artery & 3 & 4286,50 & JJ NN \\
\hline pulmonary artery & 1 & 3974,50 & JJ NN \\
\hline
\end{tabular}

Table 2: top FMA terms in radiology corpus

\section{RADLEX Terms}

Analogously, RadLex was used to identify the most relevant radiology terms. The most relevant RadLex terms are shown below. As with the FMA, the most relevant RadLex terms in the anatomy corpus are centered on "artery". In contrast, in the radiology corpus the RadLex relevance scores indeed point to a radiology profile:

\begin{tabular}{|l|r|r|r|}
\hline \multicolumn{1}{|c|}{ RadLex Term } & Freq. & Score & \multicolumn{1}{c|}{ POS } \\
\hline lateral & 464 & 338724,00 & JJ \\
\hline anterior & 452 & 314721,00 & JJ \\
\hline artery & 237 & 281961,00 & NN \\
\hline anterior spinal artery & 2 & 219894,33 & JJ JJ NN \\
\hline lateral thoracic artery & 2 & 217815,33 & JJ JJ NN \\
\hline
\end{tabular}

Table 3: top RadLex terms in anatomy corpus

\begin{tabular}{|l|r|c|r|}
\hline RadLex Term & Freq. & Score & \multicolumn{1}{l|}{ POS } \\
\hline x-ray & 253 & 81901,64 & NN \\
\hline imaging modality & 6 & 58682,00 & NN NN \\
\hline volume imaging & 1 & 57855,09 & NN NN \\
\hline molecular imaging & 4 & 57850,00 & JJ NN \\
\hline mr imaging & 9 & 57850,00 & JJ NN \\
\hline
\end{tabular}

Table 4: topRadLex terms in radiology cor pus

\section{Conclusions and Future Work}

Using ICD-9 lymphoma terminology, we will derive a Pubmed-based corpus on lymphoma to analyse the context of the statistically top most relevant terms from the FMA and RadLex terminologies. In this way we will be able to identify relationships and eventually query patterns across the three dimensions of anatomy, radiology and lymphoma research.

\section{Acknowledgments}

This research has been supported in part by the THESEUS- MEDICO Project, which is funded by the German Federal Ministry of Economics and Technology under the grant number 01MQ07016.

\section{References}

Brants T. (2000). TnT - A Statistical Part-of-Speech Tagger. In: Proc. of the 6th ANLP Conference, Seattle, WA

Langlotz, CP. (2006). RadLex: A New Method for Indexing Online Educational Materials In: Radiographics 26, pp.1595-1597.

Rosse C. and J.L.V. Mejino Jr. (2003). A reference ontology for biomedical informatics: The Foundational Model of Anatomy. Journal of Biomedical Informatics, 36(6), pp. 478-500.

Schutz A., and P Buitelaar. (2005). RelExt: A Tool forRelation Extraction in Ontology Extension In: Proc. the 4th International Semantic Web Conference, Galway, Ireland. 